\title{
AGO Recommendations for the Diagnosis and Treatment of Patients with Early Breast Cancer: Update 2015
}

\author{
Volker Hanfa Florian Schütz ${ }^{\mathrm{b}}$ Cornelia Liedtke ${ }^{\mathrm{c}}$ \\ Marc Thill ; on behalf of the AGO Breast Committee* \\ ${ }^{a}$ Frauenklinik Nathanstift, Klinikum Fürth, Germany; \\ b Universitätsfrauenklinik Heidelberg, Germany; \\ ${ }^{c}$ Klinik für Frauenheilkunde und Geburtshilfe, Universitätsklinikum Schleswig-Holstein/Campus Lübeck, Germany; \\ ${ }^{d}$ Klinik für Gynäkologie und Geburtshilfe, Agaplesion Markus Krankenhaus, Frankfurt/M., Germany
}

\author{
Keywords \\ Breast cancer - Treatment recommendations - Surgery . \\ Adjuvant treatment
}

\section{Introduction}

For the last 14 years, the Breast Committee of the Arbeitsgemeinschaft Gynäkologische Onkologie (German Gynecological Oncology Group, AGO) has been preparing and updating evidence-based recommendations for the diagnosis and treatment of patients with early and metastatic breast cancer. The AGO Breast Committee consists of gynecological oncologists specialized in breast cancer and interdisciplinary members specialized in pathology, radiological diagnostics, medical oncology, and radiation oncology. This update has been performed according to a documented rule-fixed algorithm, by thoroughly reviewing and scoring, chapter by chapter, the recent publications for their scientific validity (Oxford level of evidence (LoE), www.cebm.net) [1] and clinical

${ }^{*}$ Members of the 'AGO Breast Committee' in alphabetical order: Ute-Susann Albert, Frankfurt/M.; Ingo Bauerfeind, Landshut; Joachim Bischoff, Dessau; Jens Uwe Blohmer, Berlin; Klaus Brunnert, Osnabrück; Peter Dall, Lüneburg; Ingo J. Diel, Mannheim; Prof. Dr. Tanja Fehm, Düsseldorf; Nikos Fersis, Bayreuth; Michael Friedrich, Krefeld; Kay Friedrichs, Hamburg; Bernd Gerber, Rostock; Volker Hanf, Fürth; Nadia Harbeck, Munich; Jens Huober, Ulm; Christian Jackisch, Offenbach; Wolfgang Janni, Ulm; Hans H. Kreipe, Hanover; Sherko Kümmel, Essen; Thorsten Kühn, Esslingen; Cornelia Liedtke, Lübeck; Sibylle Loibl, Neu-Isenburg; Michael Lux, Erlangen; Hans-Joachim Lück, Hanover; Nicolai Maas, Lübeck; Volker Möbus, Frankfurt/M.; Volkmar Müller, Hamburg; Gunter von Minckwitz, Neu-Isenburg; Christoph Mundhenke, Kiel; Ulrike Nitz, Mönchengladbach; Mahdi Rezai, Düsseldorf; Achim Rody, Lübeck; Anton Scharl, Amberg; Rita Schmutzler, Cologne; Marcus Schmidt, Mainz; Andreas Schneeweiss, Heidelberg; Florian Schütz, Heidelberg; Ingrid Schreer, Kiel; H. Peter Sinn, Heidelberg. Erich Solomayer, Homburg; Rainer Souchon, Berlin; Elmar Stickeler, Freiburg i.Br.; Marc Thill, Frankfurt/M.; Christoph Thomssen, Halle/Saale; Michael Untch, Berlin; Frederik Wenz, Mannheim; Wilfried Budach, Düsseldorf (DEGRO).

The members of the AGO Breast Committee are also listed in the appendix accessible at www.karger.com $/$ ?DOI=431346 relevance (AGO grades of recommendation (GR); table 1). We present the 2015 update; the full version of the updated slide set is available online as PDF file in both English and German [2].

\section{Breast Cancer Risk Factors}

Individual risk factors can be classified into non-modifiable, modifiable, and socially defined factors. Currently, there is good evidence that changes in some modifiable risk factors could substantially decrease the individual breast cancer risk.

High total fat intake influences the body mass index (BMI) and may increase the breast cancer risk. Consequently, a healthy and lowin-fat diet should be preferred. Neither intake of fruits and vegetables nor the addition of micronutrients, trace elements, or vitamins may reduce the breast cancer risk $[3,4]$. However, prevention of diabetes mellitus type II, physical activity, and maintaining normal weight could reduce the breast cancer incidence and mortality [5].

Alcohol use may be more strongly associated with the risk of hormone-sensitive breast cancers compared to hormone-insensitive subtypes, suggesting distinct etiologic pathways [6]. In contrast, smoking is associated with overall breast cancer risk. Especially smoking at a young age (long before the first pregnancy) increases the risk by up to $60 \%$. Never smoking reduces the lifetime risk by $15-24 \%[7,8]$.

\section{Breast Cancer Risk and Prevention}

The indication of testing patients for $B R C A 1 / 2$ mutations is based on family and personal histories of breast and/or ovarian cancer. However, before genetic testing is initiated, counseling and informed consent is mandatory and should include the consequences

\section{KARGER}

Fax +497614520714

\section{(c) 2015 S. Karger GmbH, Freiburg}

$1661-3791 / 15 / 0103-0189 \$ 39.50 / 0$
Prof. Dr. med. Volker Hanf 
Table 1. AGO grades of recommendation
This investigation or therapeutic intervention is highly beneficial for patients, can be recommended without restriction, and should be performed.

$+\quad$ This investigation or therapeutic intervention is of limited benefit for patients and can be performed.

+/- This investigation or therapeutic intervention has not shown benefit for patients and may be performed only in individual cases. According to current knowledge, a general recommendation cannot be given.

This investigation or therapeutic intervention can be of disadvantage for patients and might not be performed.

-/- This investigation or therapeutic intervention is of clear disadvantage for patients and should be avoided or omitted in any case. that may follow the eventual detection of a mutation. A checklist evaluating the personal history is available in German [9]. Furthermore, $B R C A 1 / 2$ testing can be offered in patients with triple-negative breast cancer (TNBC) regardless of age ( $\mathrm{LoE} 3 \mathrm{~b} / \mathrm{C} / \mathrm{AGO}+)$, particularly if an impact on treatment decisions is anticipated. The rate of $B R C A 1 / 2$ mutations, however, has been shown to decrease with increasing age in TNBC [10]. Of all genetic testing results, $5-30 \%$ revealed variants of unknown significance (VUS). It is not yet clear whether these are causative for development of the disease. As more than $60 \%$ of the VUS are confidential or extremely rare and population specific, only large databases such as the database of the German Consortium of Hereditary Breast and Ovarian Cancer (GC-HBOC) allow for the classification of most of these VUS.

$B R C A 1$ and BRCA2 explain only about $50 \%$ of the familial aggregation. Recent data suggested that no further high-risk genes such as $B R C A 1 / 2$ (odds ratio $(\mathrm{OR})>5.0$ ) exist and that the remaining heritability is due to moderate-risk genes (e.g. RAD51C, ATM, BRIP1, CDH1, CHEK2, NBN, PALB2, PTEN; OR 1.5-5.0) and lowrisk variants (FGFR2, TOX3, 2q35, 11q15, SLC4A7, 5p12, MAP3K1; $\mathrm{OR}<1.5)$ that are transmitted via an oligogenetic trait $[11,12]$. Low-risk variants also showed associations with specific breast cancer subtypes. RAD51C and PALB2 were recently described as moderate-to-high-risk genes [13]. Moreover, there are many non$B R C A$-associated hereditary cancer syndromes with an increased risk for breast cancer (LiFraumeni, Cowden, hereditary diffuse gastric cancer syndrome, Peutz-Jeghers syndrome, Lynch syndrome). The use of commercially available but not validated breast cancer gene panels for risk prediction is not recommended outside of controlled clinical trials. Furthermore, clinical genetic testing for lowrisk variants should be avoided (LoE 3b/D/AGO -). For many of these genetically defined subtypes, issues such as histopathological features, sensitivity to different screening modalities, course of disease, or specific treatment response still remain unclear. Healthy women who are identified as being at moderate to high risk for disease development should be offered the participation in a surveillance/screening program for the detection of early-stage breast cancer ( $\operatorname{LoE} 2 \mathrm{a} / \mathrm{B} / \mathrm{AGO}++)$. Women with BRCA1/2 mutations should also be offered nondirective counseling for the uptake of primary preventive measures (e.g. risk-reducing bilateral salpingooophorectomy at around 40 years of age $(\mathrm{LoE} 2 \mathrm{a} / \mathrm{B} / \mathrm{AGO}++)$, riskreducing bilateral mastectomy ( $\operatorname{LoE} 2 \mathrm{a} / \mathrm{B} / \mathrm{AGO}+)$, or medical prevention with tamoxifen $(\mathrm{LoE} 1 \mathrm{a} / \mathrm{A} / \mathrm{AGO}+)$, raloxifen $(\mathrm{LoE} 1 \mathrm{~b} / \mathrm{A} /$ $\mathrm{AGO}+)$, or an aromatase inhibitor $(\mathrm{AI})(\mathrm{LoE} 1 \mathrm{~b} / \mathrm{A} / \mathrm{AGO}+)$ ), in addition to participation in a surveillance/screening program. However, unilateral or bilateral mastectomy is not indicated in the absence of clearly defined genetic risk factors ( $\operatorname{LoE} 2 \mathrm{a} / \mathrm{B} / \mathrm{AGO}+$ ).
To date, there are no treatment recommendations specific to diseased mutation carriers. Breast-conserving surgery (BCS) is safe $(\mathrm{LoE} 2 \mathrm{a} / \mathrm{B} / \mathrm{AGO}+)$ and systemic therapy can be given according to recommendations for sporadic breast cancer ( $\mathrm{LoE} 3 \mathrm{a} / \mathrm{B} / \mathrm{AGO}+$ ). Additionally, carboplatin represents a treatment option used in favor of docetaxel in metastatic BRCA-mutated breast cancer [14] $(\mathrm{LoE} 2 \mathrm{ba} / \mathrm{B} / \mathrm{AGO}+)$. The use of poly(ADP-ribose) polymerase (PARP) inhibitors is currently being validated in prospective studies (LoE 2b/D/AGO +/-).

\section{Breast Cancer Diagnostics}

Early detection and screening of breast cancer aims to reduce the breast cancer-specific mortality and treatment-dependent morbidity. Detection of invasive breast cancer at an early stage (stage I-IIA) offers the chance to survive this disease with less treatment impairment and better quality of life. Professionals and women need to be informed about the benefits and harms of cancer screening tests before making medical decisions. This includes clear and understandable information in absolute terms about false-positive rates, falsenegative rates, overdiagnosis, and overtreatment. Contrary to persistent criticism (mostly based on the complexity of data analysis), all available evidence confirms that mammography screening is capable of significantly reducing breast cancer mortality. The limitations of mammography are well known so that new technologies to overcome these constraints are highly welcome. Within 2 randomized controlled trials and further cohort studies, digital breast tomosynthesis (DBT) proved to significantly increase both sensitivity and specificity, thus improving diagnostic accuracy. In the near future, DBT can be expected to replace standard 2-dimensional (2D) mammography for mammography screening [15]. At present, DBT can be recommended if further mammographic diagnostics like a digital spot compression view is requested [16] (LoE 2b/B/AGO +).

There are no data to recommend breast ultrasound (US) alone or automated breast volume scanning (ABVS) as breast cancer screening methods. A recent Cochrane Database Systematic Review from 2013 did not detect any completed controlled studies on the use of adjunct ultrasonography for screening in women at average risk for breast cancer. Solely, 1 ongoing randomized controlled trial was identified. The arguments against US used as a screening modality alone are lack of reproducibility, high false-positive rates, low positive predictive value (PPV) for biopsy, the inability to detect most ductal carcinoma in situ (DCIS) cases, operator dependency, and lack of quality assurance. That is why 'presently, there is no methodologically sound evidence available, justifying the rou- 
tine use of ultrasonography as an adjunct screening tool in women at average risk for breast cancer' [17] ( $\mathrm{LoE} 3 \mathrm{~b} / \mathrm{C} / \mathrm{AGO}-$ ).

The results of recently published cohort studies with automated whole-breast US examination (ABUS/ABVS) promise to reduce the physician examination time and operator dependency. Further research is necessary to demonstrate equal accuracy as with standard hand-held US examination [18] (LoE 3b/B/AGO +/-).

Elastography is an evolving new US-based imaging technology and has some advantages in diminishing the rate of Breast Imaging Report and Data System (BI-RADS) group III lesions and in measuring the true size of breast cancer lesions. To further characterize focal breast lesions in addition to B-mode sonography in order to differentiate between BI-RADS 3 and 4a masses, ultrasonographic elastography helps to decrease the false-positive biopsy rate [19] ( $\mathrm{LoE} 2 \mathrm{~b} / \mathrm{B} / \mathrm{AGO}+)$.

Contrast-enhanced magnetic resonance imaging (MRI) plays an important role in acknowledged indications in diagnostic breast imaging as well as in high-risk patients. MRI should not be used generally to assess symptoms or breast lesions. MRI can, however, be used if clinical examination, mammography and sonography do not allow a definite diagnosis (LoE $2 \mathrm{~b} / \mathrm{B} / \mathrm{AGO}+/-$ ).

MRI should not be used generally for preoperative staging purposes in case of breast-conserving treatment. According to a metaanalysis, the re-excision rate is not reduced, but the initial and total rates of mastectomy are increased if a preoperative breast MRI is performed compared with no preoperative breast MRI. In case of lobular invasive breast cancer, there is a significant reduction of the re-excision rate and no significant impact on the rate of mastectomies due to the use of preoperative breast MRI. Furthermore, the preoperative breast MRI does not reduce the rate of local recurrences and does not improve the local recurrence-free survival and the distant metastasis-free survival [20]. This is why preoperative breast MRI is not recommended as a routine method for all patients. For some patients, e.g. with high breast density (American College of Radiology (ACR) categories 3-4), lobular invasive cancer, suspicion of multilocular disease and high risk, it may be considered (LoE 1b/B/AGO +/-). MRI-guided vacuum biopsy is mandatory in case of MRI-detected additional lesions.

If there is a clinically and/or sonographically suspicious axillary lymph node, elastography adds to diagnostic accuracy [21]. USguided fine-needle aspiration or core cut biopsy is recommended to avoid 2-stage axillary surgery [22] ( $\mathrm{LoE} 2 \mathrm{~b} / \mathrm{B} / \mathrm{AGO}+)$. The standard procedure in patients with unsuspicious axillary lymph nodes is sentinel node biopsy.

\section{Pathology}

Regarding conventional histopathology, the effect of pre-analytic factors is not only important to accurately evaluate lymphatic invasion and distinguish it from retraction artefacts [23] but also to preserve antigen fixation. This concerns the cold ischemia time and the duration of fixation [24], and is of particular importance for human epidermal growth factor receptor 2 (HER2) immunohistochemistry (IHC) [25]. The relevance of the tumor size and the methods for its determination have been reviewed recently [26], and in this context it is emphasized that tumor size, multifocality, and multicentricity are important prognostic parameters [27]. This should also be considered in tumor/node/metastasis (TNM) staging of breast cancer [28]. The role of peritumoral lymphovascular invasion for prognosis was also reviewed and re-assessed in 2014, and the relevance of estimating its extent was emphasized [31]. However, venous invasion (V0/V1) is very rare (1\%), and its independent prognostic significance is not clearly established. Therefore, the differentiation between venous invasion and lymphovascular invasion is considered optional. The standardized reporting of tumorinfiltrating lymphocytes [32] is a new recommendation that is based on studies demonstrating its prognostic and predictive value in randomized adjuvant and neoadjuvant clinical trials, particularly with regard to platinum-based chemotherapy [33]. Lymphocyte infiltration of the tumor stroma should be reported as a percentage parameter in the pathologic report when there is a predominant lymphocytic infiltrate (> 50\%). The pathology reporting of tumor regression after neoadjuvant chemotherapy (NACT) has been reviewed [34] and should include the estimation of residual tumor proliferation as a predictor of the further clinical course of the patient [35]. However, a particular regression score, such as the residual cancer burden estimator [36], was not agreed on in this guideline.

With regard to determination of the estrogen receptor (ER), the threshold of $1 \%$ for reporting the tumor as being ER positive remains, but it is recognized that the low ER-expressing tumors should be regarded as a separate group and may be biologically similar to hormone-negative breast cancers [37]. The progesterone receptor $(\mathrm{PgR})$ is an independent prognostic indicator [38]; however, in the absence of detectable ER levels, expression of the PgR is a rare event. This ER-negative/PgR-positive subgroup is often attributable to technical issues leading to a false-negative ER or false-positive PgR status, due to the high sensitivity of the current IHC systems [39]. Therefore, a positive PgR status should be reported with a higher threshold of $10 \%$ compared to $1 \%$ for the ER. Gene expression studies regarding the ER/PgR status have shown that the molecular analysis is not only highly consistent with the IHC results, but also equivalent or better for predicting endocrine responsiveness [40]. Therefore, the option of evaluating hormone receptors using validated gene expression test kits has been included in these guidelines. RNA sequencing is an alternative method for gene expression studies in breast cancer [41], but no robust data have so far been published to determine its value in clinical practice. Therefore, RNA sequencing is not recommended as a routine method for the evaluation of hormone receptors. With regard to the molecular analysis of the HER2 amplification status, only in situ hybridization (ISH) can be considered a clinically valid method and is recommended as the basis of therapy decisions [42]. Based on encouraging results from the molecular determination of HER2 in clinical trials, the evaluation of HER2 by employing validated gene expression test kits has been mentioned in these guidelines. The evaluation of the Ki-67 score should follow recommendations regarding its reproducibility [43], which may be further improved by the use of image analysis.

Last but not least, quality control measures are crucial for all 
parts of pathology. It has been shown recently that a second review of the breast pathology identifies clinically significant discrepancies in over $10 \%$ of the patients. Therefore, adequate quality control must be instituted in IHC, in the determination of the HER2-status, and in the reporting of breast cancer [44].

\section{Lesions of Uncertain Malignant Potential (B3)}

According to the European Guidelines for Quality Assurance in Breast Cancer Screening and Diagnosis, non-malignant, but atypical, or indeterminate breast lesions detected on core needle biopsy are classified as B3 [45]. Lesions of uncertain malignant potential (B3) exhibit varying risks of associated malignancy, which might not be represented in core needle biopsies. The definitions, classification, and therapeutic implications of B3 lesions, as defined by the AGO recommendations, have been described recently [46].

In the 2015 issue of the B3 chapter, changes have been introduced only with regard to intraductal papilloma. Formerly, core needlebased diagnosis of these intraductal proliferations was reported to exhibit a PPV for malignancy of up to $20 \%$ [47]. The more papillomas were discovered by mammography screening, the more it became evident that this figure might represent an overestimation and that the PPV is far lower. In 2013 and 2014, 3 studies were published providing evidence that patients with representative (at least $100 \mathrm{~mm}^{2}$ or 7 12-g cores need to be evaluated [48]) core needle biopsy-based diagnosis of intraductal central papilloma may safely be spared from open biopsy [48-50]. Therefore, it appears justified to consider papilloma rather as a B2 lesion not requiring further surgery rather than as a true B3 lesion. This is, however, unless atypia is present [51].

Besides nuclear pleomorphism, atypia becomes obvious from rarefication of myoepithelial cells in papilloma [51]. When there are signs of atypia ('atypical papilloma'), open biopsy is warranted (fig. 1,www.karger.com/?DOI=431346).

\section{Prognostic and Predictive Factors in Early Breast Cancer}

In node-negative breast cancer, the urokinase-type plasminogen activator (uPA)/plasminogen activator inhibitor-1 (PAI-1) enzyme-linked immunosorbent assay (ELISA) $\left(\right.$ Femtelle $\left.^{\circledR}\right)$ is a recommended ( $\operatorname{LoE} 1 \mathrm{a} / \mathrm{A} / \mathrm{AGO}+$ ) prognostic and predictive factor for the decision regarding adjuvant chemotherapy [52]. This option remains unchanged compared to previous years, despite its limited feasibility due to the need for fresh frozen tissue samples.

In doubtful cases of node-negative, endocrine-responsive, HER2negative tumors, a multigene assay such as Oncotype DX ${ }^{\circledR}$, Endopredict ${ }^{\circledR}$ or Prosigna ${ }^{\circledR}$ [53] may be helpful in the decision-making process to opt for endocrine therapy alone versus a chemoendocrine treatment option (LoE $2009 \mathrm{I} / \mathrm{B} / \mathrm{AGO}+)$. All 3 tests are now equally recommended by the AGO Breast Committee under the given clinical restraints. In node-positive patients, the use of these assays needs to be carefully discussed with the patient (LoE 2009 II/B/AGO +/-).
Furthermore, all 3 tests, Oncotype DX ${ }^{\circledR}$, Endopredict ${ }^{\circledR}$, and Prosigna ${ }^{\circledR}[54]$, now provide information about late recurrences, which may be used to guide endocrine therapy for more than 5 years.

Circulating tumor cells (CTCs; Cell Search ${ }^{\circledR}$ ) should be used only in the metastatic setting as a prognostic (LoE 2009 I/B/AGO + ) and early treatment monitoring tool.

\section{Neoadjuvant Chemotherapy}

Survival rates are similar after primary systemic ('preoperative', 'neoadjuvant') therapy and adjuvant therapy [55]. Pathological complete response (pCR) defined as ypT0 ypN0 or ypT0/is ypN0 is associated with improved survival [56].

NACT is indicated in every case with an indication for adjuvant postoperative chemotherapy (LoE 1/B/AGO +) [57]. In particular, in patient subgroups where a $\mathrm{PCR}$ is associated with improved survival such as in triple-negative and HER2-positive cancers, NACT (plus targeted therapy) should be the preferred therapeutic approach (AGO $++)[56,58]$. In patients with TNBC and a BRCA1/2 mutation or positive family history for breast or ovarian cancer according to the German guidelines, a platinum salt-containing regimen may be considered ( $\mathrm{LoE} 2 \mathrm{~b} / \mathrm{B} / \mathrm{AGO}+$ ) based on data from phase II randomized trials (e.g. GeparSixto, Cancer and Leukemia Group B (CALGB) 40603) and an exploratory retrospective analysis of GeparSixto [59-63].

Response-guided treatment has been shown to be beneficial within the GeparTrio trial. Consequently, in case of response after 2 cycles of docetaxel/adriamycin/cyclophosphamide (DAC) in hormone receptor-positive breast cancer, a total of 8 instead of 6 cycles of DAC may be considered ( $\mathrm{LoE} 2 \mathrm{~b} / \mathrm{C} / \mathrm{AGO}+$ ). In the case of no response after 2 cycles of DAC, continuation of neoadjuvant systemic therapy with a non-cross-resistant regimen ( $\mathrm{LoE} 2 \mathrm{~b} / \mathrm{B} / \mathrm{AGO}$ + ), such as $4 \times$ vinorelbine/capecitabine (NX) $(\mathrm{LoE}$ 1b/B/AGO +) may be beneficial $[64,65]$. This can be an option in individual cases but cannot be considered as a routine approach.

With respect to endocrine neoadjuvant therapy, in exceptional situations, endocrine treatment with luteinizing hormone-releasing hormone (LHRH) plus AI may be considered for premenopausal women ( $\mathrm{LoE} 1 \mathrm{~b} / \mathrm{C} / \mathrm{AGO}+/-)$. Novel predictive factors, such as tumor cell infiltration/lymphocyte-predominant breast cancer (LoE I/B/AGO +/-) or phosphatidylinositol-4,5-bisphosphate 3-kinase catalytic subunit alpha (PIK3CA) mutation in the tumor (LoE $\mathrm{II} / \mathrm{B} / \mathrm{AGO}+/-$ ), are promising tools but not yet applicable to the routine clinical setting $[66,67]$.

The indications for mastectomy after NACT remain unchanged: positive margins after repeated excisions ( $\mathrm{LoE} 3 \mathrm{~b} / \mathrm{C} / \mathrm{AGO}++$ ), lack of feasibility of radiotherapy ( $\mathrm{LoE} 5 / \mathrm{D} / \mathrm{AGO}++$ ), and presence of inflammatory breast cancer (in case of no more than clinical complete response, LoE $2 \mathrm{~b} / \mathrm{C} / \mathrm{AGO}+$ ). In inflammatory breast cancer with pCR after NACT, BCS may be discussed with the patient. Furthermore, the presence of large tumors $(\mathrm{cT} 4 \mathrm{a}-\mathrm{c})$ represents only a relative indication for mastectomy after NACT (LoE 2b/B/AGO $+/-)$. Multicentric lesions should be exactly defined (bicentric, tricentric, $\mathrm{AGO}+/-)$ as an individual option, considering the scarce 
database for such an approach. Similarly, the remaining tumor load after NACT must be weighed up against breast size (LoE 2b/C/AGO +/-) [68]. Post-neoadjuvant concepts are currently investigated in clinical trials. There is at present no indication for further chemotherapy in case of no pCR.

\section{Breast Cancer Surgery under Oncological Aspects}

Breast surgery represents just one out of multiple steps of breast cancer treatment. Thus, both a diagnostic and an oncological expertise are indispensable and a definite requirement $(\mathrm{AGO}++)$.

In the past year, no practice-changing data concerning surgery were presented. Subsequently, only minor modifications have been made in the 2015 AGO recommendations. A tumor-free margin for an invasive breast cancer is now defined with 'no cells on ink'. No other definition should be used in case of unfavorable tumor biology: There is no need to call for a particular excision margin in more aggressive subtypes, such as the triple-negative subtype (LoE 2a/B/AGO ++) [69].

One possible technique to lower the re-resection rate is USguided surgery, which can be recommended in breast cancer that is visible in US or palpable (LoE 1a/A/AGO +/-). 2 new meta-analyses have confirmed an advantage for US guidance as compared to palpation or wire marking $[70,71]$.

Despite the ongoing intense discussion regarding surgical axillary management, sentinel lymph node biopsy (SLNB) is widely accepted as the 'gold standard' and has replaced axillary lymph node dissection (ALND) as a staging procedure for early node-negative breast cancer. The available evidence suggests that quality-assured SLNB is a reliable predictor of the axillary lymph node status, with high levels of sensitivity (90-95\%), specificity (100\%), negative predictive value (NPV) (95\%), and accuracy (97\%) [72]. In patients treated with NACT, the histopathological response (which includes response in breast and lymph nodes) is an important prognostic factor. Therefore, it would seem more reasonable to perform SLNB after NACT in order to provide this important prognostic factor and decide on the necessity of a post-neoadjuvant treatment in case of a missing pathological complete remission. SLNB after NACT, however, is associated with less favorable success rates (such as detection and false-negative rate) as compared to SLNB in cases of a primarily surgical approach $[73,74]$. Although limited new data were published last year $[75,76]$, we found no justification to change our previous year's recommendation (LoE 2a/B/AGO +/-; fig. 2, www.karger.com/?DOI=431346).

Patients with suspicious lymph nodes before NACT may receive diagnostic core needle or fine-needle biopsy in order to confirm axillary involvement cytologically/histologically prior to initiation of chemotherapy (LoE 2a/B/AGO +) [22]. Proven axillary lymph node involvement (needle biopsy or SLNB) is an indication for ALND following NACT.

In case of positive SLNB before NACT (fig. 2, www.karger. com/?DOI=431346), we recommend that ALND may only be omitted in cases meeting the American College of Surgeons Oncology Group (ACOSOG) Z011 criteria, i.e. cT1/2 and cN0 tumors, positivity of $<3$ lymph nodes, lack of extracapsular spread, anticipation of whole- breast irradiation (WBI) for BCS, and administration of adequate systemic therapy (LoE 1a/B/AGO +/-). We are eagerly looking forward to the start of the Intergroup Sentinel Mamma (INSEMA) trial, which is felt to shed some more light on optimization of axillary surgery.

In recent years, intensive research was done to develop highly accurate methods and techniques that use radiotracer-free marking techniques for SLNB. At present, the radiotracer/gamma probe technique with the $99 \mathrm{mTc}$ colloid is the most accurate standalone method for SLNB (LoE 1a/A/AGO ++). However, some inherent disadvantages of this technique exist, like the exposure of patient and healthcare personnel to radiation, limited tracer availability, and a short half-life. Moreover, after the use of blue dye, intensive tattoos may be seen on the breast for several months and anaphylactic reactions related to the blue dye have already occurred and may be lifethreatening. Thus, other techniques using, e.g., indocyanin green (ICG) (LoE 2b/B/AGO +/-) and superparamagnetic iron oxides (SPIO) (LoE $2 \mathrm{~b} / \mathrm{B} / \mathrm{AGO}+/-)$ have been examined with an increasing body of evidence. The results of studies with SPIO [77] and ICG [78] showed an equivalent lymph node identification rate, detection rate, and concordance rate when compared with the radiotracer.

\section{Adjuvant Radiotherapy}

In most recommendations, the task forces of the AGO and the German Society of Radiation Oncology (DEGRO) have a broad agreement on adjuvant locoregional radiotherapy of patients with early breast cancer. Nevertheless, several issues remain partially controversial. In 2014, divergent recommendations were issued by the AGO and the DEGRO for specific clinical situations (e.g. the role of hypofractionated (HF) WBI). In 2015, a joint task force with 3 members of the AGO and 3 members of the DEGRO was founded to define a common consensus between the two societies. For most issues a consensus was reached. The few remaining controversial aspects regarding the clinical implications of current evidence are highlighted.

While post-mastectomy radiotherapy (PMRT) for patients with more than 4 involved axillary nodes is unequivocally recommended, new data from a meta-analysis of the Early Breast Cancer Trialists' Collaborative Group (EBCTCG) have been published in 2014 [79]. These data confirm the lack of benefit of PMRT in nodenegative patients. In women with 1-3 involved lymph nodes, radiotherapy reduces the locoregional recurrence $(2 \mathrm{p}<0.00001)$, overall recurrence (recurrence rate $(\mathrm{RR}) 0.8,2 \mathrm{p}=0.00006$, and breast cancer mortality (RR $0.8,2 \mathrm{p}=0.01)$.

The DEGRO therefore recommends PMRT in all node-positive patients $(\mathrm{LoE} 1 \mathrm{a} / \mathrm{AGO}+$ ), whereas the AGO points out the limited relevance and validity of this meta-analysis for today's clinical practice. Patients, who entered the meta-analysis were accrued between 1964 and 1986 and underwent less modern systemic treatments (e.g. no tamoxifen in premenopausal patients, no taxanes, no trastuzumab, etc.). The reported RRs (40-50\% RR for patients with 1-3 positive nodes and $70-80 \%$ for patients with 4 and more involved nodes) do not reflect the patient outcome in a modern clinical setting. Considering the important impact of radiotherapy on breast 
reconstruction, the AGO recommends to stratify the PMRT indication by morphological and biological risk factors. There is consensus between the AGO and the DEGRO regarding the indication for PMRT in patients with high-risk features. For women with 1-3 positive nodes and low-risk tumors, the AGO does not identify a benefit of PMRT for the individual patient (LoE 5/D/AGO +/-).

WBI remains the standard of care for patients who undergo breast-conserving therapy. In elderly patients ( $>70$ years), in case of ER-positive, grade 1-2, HER2-negative tumors of up to $3 \mathrm{~cm}$, if adjuvant endocrine treatment (e.g. tamoxifen for 5 years) is reliably performed, breast irradiation is able to reduce the risk of local recurrence by about $8 \%$ over 10 years [80]. There was no impact on the secondary mastectomy rate, the metastasis-free survival or on overall survival (OS) in this study. Elderly patients should be advised on the following: In older patients with pT1-2 $(=<3 \mathrm{~cm})$ pN0 hormone receptor-positive breast cancer, breast irradiation for breast-conserving therapy is able to reduce the risk of local recurrence by about $8 \%$ over 10 years. A benefit with regard to metastasis-free survival and OS has not been found yet.

In 2015, a consensus was achieved between the AGO and the DEGRO regarding the role of HF regimens for WBI. 5 randomized trials did not show any inferiority of HF regimens compared to conventionally fractionated schedules. In one of the trials, there were significantly fewer distant relapses in up to 10 years in the 40 -Gy group (hazard ratio (HR) $0.74,95 \%$ confidence interval (CI) 0.59-0.94), which contributed to the significantly higher rates of disease-free survival (DFS) and OS in the 40-Gy group compared with the 50-Gy group. (HR $(\mathrm{OS})=0.8 ; \mathrm{p}=0.042)$ [81]. Some of the normal-tissue effects were less common after the 15 -fraction regimen than after the conventional schedule (breast shrinkage, telangiectasia, breast edema). Due to some concerns about the long-term outcome after a HF regimen in younger patients and women with a higher biological risk, the AGO/DEGRO consensus recommended an age- and risk-adapted concept for WBI. Boost radiotherapy is generally recommended for patients $<60$ years. For older women, the absolute effect is small and the benefit doubtful. Partial breast irradiation was not recommended as a sole radiotherapy modality.

Radiotherapy to the axillary lymph nodes is indicated in patients with tumor residuals in the axilla ( $\mathrm{LoE} 5 / \mathrm{D} / \mathrm{AGO}++)$. In patients who underwent BCS and had 1-2 positive sentinel lymph nodes (if the ACOSOG Z011 criteria are met), it is open whether axillary dissection, radiotherapy or no further axillary treatment is the best option ( $\mathrm{LoE} 1 \mathrm{~b} / \mathrm{B} / \mathrm{AGO}+/-$ ); this is similar in patients after mastectomy with 1-2 positive sentinel lymph nodes and if the ACOSOG Z011 criteria are met but on an inferior level of evidence (LoE 5/AGO +/-). In patients with BCS or mastectomy and positive lymph nodes and with the ACOSOG Z011 criteria not met (or chest wall irradiation not planned), axillary dissection or radiotherapy should be performed (LoE 1b/B/AGO ++) (fig. 3, www.karger. $\mathrm{com} /$ ?DOI=431346). In patients with $>3$ positive nodes, further treatment of the axilla is recommended, either by axillary node dissection ( $\operatorname{LoE} 1 \mathrm{~b} / \mathrm{B} / \mathrm{AGO}++$ ) or by axillary radiotherapy (LoE 1b/B/ $\mathrm{AGO}+$ ). Regarding the radiotherapy of other locoregional lymph node areas, 2 randomized (yet unpublished) trials demonstrated an improved DFS and distant DFS for patients who had received radiotherapy to the supraclavicular and internal mammary nodes $[82,83]$. There was consensus between the DEGRO and AGO to recommend irradiation of the extra-axillary lymph node areas in patients with positive axillary lymph nodes and high-risk features $(\mathrm{LoE} 2 \mathrm{~b} / \mathrm{B} / \mathrm{AGO}+)$. In contrast to the DEGRO, the AGO does not identify a benefit in low-risk patients ( $\mathrm{LoE} 2 \mathrm{~b} / \mathrm{B} / \mathrm{AGO}+/-$ ).

\section{Adjuvant Endocrine Therapy}

Endocrine therapy represents the most important option in the adjuvant treatment of early estrogen-responsive breast cancer. Treatment decisions for this targeted therapy are based on the proof of endocrine sensitivity (ES) by well-established predictive factors. ES, by definition, requires staining positivity of the tumor cells of at least $1 \%$ for either the ER or the PgR. For the determination of the menopausal status, measurement of estradiol (E2) and follicle-stimulating hormone (FSH) represents the method of choice.

Acknowledging data with regard to the integration of extended adjuvant treatment (EAT) strategies using (i) tamoxifen (as shown by the ATLAS [84] and aTTom [85] trials) or (ii) AIs (as demonstrated for instance in the MA.17 trial [86]), the following general recommendations can be given for the adjuvant endocrine therapy $(\mathrm{AGO}++)$ :

- standard treatment duration 5 years: tamoxifen in premenopausal, tamoxifen or $\mathrm{AI}$ in postmenopausal patients

- treatment for up to 10 years may be considered based on the individual risk of relapse (e.g., $\mathrm{N}+$ status)

- premenopausal: after 5 years of tamoxifen; EAT: a further 5 years of tamoxifen

- postmenopausal: after 5 years of tamoxifen; EAT: a further 5 years of tamoxifen or AI

- duration, choice and sequence of AI or tamoxifen mainly depend on the menopausal status and side effects

- switching to another endocrine treatment (tamoxifen or AI) is better than to stop

- AI as first treatment preferably in postmenopausal patients at high risk and or with lobular cancers

- no evidence for $\mathrm{AI}>5$ years

In premenopausal women, administration of tamoxifen for $5-10$ years is the standard regimen ( $\mathrm{LoE} 1 \mathrm{a} / \mathrm{A} / \mathrm{AGO}++)$. Treatment should be performed as long as it is tolerable and the patients stay premenopausal. A switch to an AI when the patients become postmenopausal may be considered as well as a prolongation of therapy up to 10 years with tamoxifen.

The TEXT and SOFT trials investigated the effect of ovarian function suppression (OFS) in the context of adjuvant endocrine therapy for women with premenopausal breast cancer. Application of gonadotropin-releasing hormone $(\mathrm{GnRH})$ in combination with tamoxifen or exemestane was compared to tamoxifen monotherapy [87]. While in TEXT all patients were treated with GnRH from the beginning, in SOFT, only those patients with premenopausal status at 8 months after completion of chemotherapy were allowed to enter the trial. Due to low event rates, a joint analysis of the TEXT and 
SOFT trials comparing tamoxifen versus OFS + tamoxifen versus OFS + exemestane was performed. Adding OFS to tamoxifen did not provide a significant benefit in the overall study population. However, there was a difference in the OFS + tamoxifen versus the OFS + exemestan groups. After a median follow-up of 68 months, the DFS at 5 years was $91.1 \%$ in the exemestane/OFS group and $87.3 \%$ in the tamoxifen/OFS group (HR for disease recurrence, second invasive cancer, or death, $0.72 ; 95 \%$ CI $0.60-0.85 ; \mathrm{p}<0.001$ ). With 194 deaths (4.1\% of the patients), the OS did not differ significantly between the two groups (HR for death in the exemestane/OFS group, 1.14; 95\% CI 0.86-1.51; $\mathrm{p}=0.37$ ) [87]. An additional retrospective exploratory analysis of the SOFT trial indicates that the major effect was seen in younger patients under 35 years of age, who received adjuvant chemotherapy due to higher risks such as highgrade invasive breast cancer and lymph node positivity, with an improvement in DFS (67\% for the tamoxifen only group vs. $78.9 \%$ for OFS + tamoxifen vs. $83.4 \%$ for OFS + exemestane). Therefore, the addition of OFS to endocrine treatment may be considered in patients with persistent ovarian function (within 8 months) after adjuvant chemotherapy: OFS 5 years + tamoxifen 5 years (LoE 1b/B/ $\mathrm{AGO}+/-)$ or OFS 5 years + AI 5 years $(\mathrm{LoE}$ 1b/B/AGO +/-). How ever, increased side effects may impair the patients' compliance.

For postmenopausal patients, the following treatment options are available:

- sequential therapy for 5-10 years (AGO ++)

- tamoxifen followed by AI (2-5 years) (LoE 1a/A)

- AI (2-5 years) followed by tamoxifen (LoE 1b/C, which may

be used preferentially in patients with node-positive disease)

- 5-10 years of tamoxifen ( $\mathrm{LoE} 1 \mathrm{a} / \mathrm{A} / \mathrm{AGO}++$ )

-5 years of AI (which may be used preferentially in high-risk and lobular cancers)

\section{Adjuvant Cytotoxic and Targeted Therapy}

In case of indication of chemotherapy, neoadjuvant therapy should always be considered $(\mathrm{AGO}++)$.

In adjuvant therapy, systemic treatment encompassing 6 cycles of fluorouracil/epirubicin/cyclophosphamide (FEC) is no longer recommended. Comparison of regimens using 4 cycles of doxorubicin and cyclophosphamide (AC) with 6 cycles of FEC in the framework of the National Surgical Adjuvant Breast and Bowel Project (NSABP) B-36 trial showed no differences neither in the DFS nor in the OS after a median follow-up period of 82.8 months ( $\mathrm{p}=0.74$ and $\mathrm{p}=0.65$, respectively) [88]. Analysis of the hormone receptor status also showed no advantage for 6 cycles of FEC. However, the side effects with FEC were much more severe and more deaths occurred as compared to 4 cycles of AC (5 vs. 2; fig. 4, www.karger.com/?DOI=431346).

Standard adjuvant chemotherapy consists of combination regimens based on anthracyclines and taxanes in patients with a HER2negative tumor $(\mathrm{LoE} 1 \mathrm{a} / \mathrm{A} / \mathrm{AGO}++)$ [3]. Treatments of choice include $4 \times$ epirubicin/cyclophosphamide (EC)/AC, followed by $12 \times$ paclitaxel (q7d), or $4 \times$ docetaxel (q21d) or treatment with $6 \times$ docetaxel/doxorubicin/cyclophosphamide (DAC; AGO ++). These combination regimens have recently been confirmed using the 10year data from E1199 [89]. Here, regimens with 3-weekly docetaxel $\left(100 \mathrm{mg} / \mathrm{m}^{2}\right.$ body surface area (BSA) q21d), weekly docetaxel (35 $\mathrm{mg} / \mathrm{m}^{2}$ BSA q7d), 3-weekly paclitaxel (175 mg/m² BSA q21d), or weekly paclitaxel $\left(80 \mathrm{mg} / \mathrm{m}^{2}\right.$ BSA q7d) were compared after 4 cycles of AC. The study population was enriched with patients carrying high-risk features (46\% premenopausal, only $12 \%$ with negative nodal status). There were significant advantages for weekly paclitaxel and docetaxel given every 3 weeks regarding the DFS, but not the OS (DFS p < 0.001, OS p = 0.07). Therefore, 3-weekly docetaxel in particular was found to be more effective than 3-weekly paclitaxel (DFS: HR 0.79, 95\% CI 0.68-0.90; OS: HR 0.86, 95\% CI $0.73-$ $1.00)$ and weekly paclitaxel was more effective than 3-weekly paclitaxel (DFS: HR 0.84, 95\% CI 0.73-0.96; OS: HR 0.87, 95\% CI $0.75-$ 1.02). Of particular interest was the subgroup with TNBC: After 4 cycles of AC, weekly paclitaxel showed a significant OS benefit in comparison with 3-weekly paclitaxel, q21d (HR 0.69, 95\% CI 0.500.94). This treatment should be considered in patients with TNBC.

If comorbidities forbid the use of anthracyclines, treatment with docetaxel and cyclophosphamide represents an alternative (LoE $\mathrm{b} / \mathrm{B} / \mathrm{AGO}+)$. In individual cases, treatments using paclitaxel mono weekly (LoE 1b/B/AGO +/-) or cyclophosphamide/methotrexate/fluorouracil (CMF) (LoE 1a/A/AGO +/-) may also be considered $[90,91]$. In case of high tumor burden - e.g., with 4 or more affected lymph nodes - dose-dense and dose-escalated treatment with epirubicin followed by paclitaxel followed by cyclophosphamide, q14d, should be considered instead of the standard regimen. Chemotherapy with dose-dense EC (q2w, x4) followed by Paclitaxel ( $\mathrm{q} 2 \mathrm{w}, \mathrm{x} 4$ ) as evaluated in the GIM2 study may represent an alternative option [127]. At present, platinum cannot be recommended in the adjuvant setting due to lack of data and should be considered for individual cases ( $\mathrm{LoE} 5 / \mathrm{D} / \mathrm{AGO}+$ ). This is in contrast to recommendations regarding the neoadjuvant setting (see above).

In HER2-positive disease, a combination using trastuzumab starting simultaneously with the taxane is recommended. The optimal duration is 1 year (LoE $1 \mathrm{~b} / \mathrm{A} / \mathrm{AGO}++)$ [92]. In individual cases - e.g., when comorbidities or side effects inhibit longer treatment treatment for 6 months may be considered (LoE 1b/A/AGO +/-) [93]. Alternative anthracycline-free combination partners for trastuzumab are docetaxel and carboplatin ( $\mathrm{LoE} 1 \mathrm{~b} / \mathrm{GR} \mathrm{A} / \mathrm{AGO}+$ ) or in individual cases - e.g., in patients with tumors $<3 \mathrm{~cm}$ and negative nodal status [94] - treatment with $12 \times$ paclitaxel (q7d, LoE $2 \mathrm{~b} / \mathrm{B} / \mathrm{AGO}+/-)$ [95]. With regard to alternative anti-HER2 treatments, neither lapatinib nor dual therapy with lapatinib + trastuzumab can currently be recommended ( $\operatorname{LoE} 1 b^{\mathrm{a}} / \mathrm{B} / \mathrm{AGO}-$ ). The results of the ALTTO study showed no advantage regarding DFS or OS neither for the sequence of trastuzumab and lapatinib nor for the combination [94]. However, in the subgroup of hormone receptor-negative patients, a trend towards improved DFS was observed for the combination (HR 0.82, 95\% CI 0.65-1.04). It is possible that this group may benefit, but the current state of data is insufficient for a recommendation. With regard to dual therapy using trastuzumab + pertuzumab, the evaluation of ongoing studies is awaited. 


\section{Gynecological Issues in Breast Cancer Patients}

This is a new chapter covering the treatment of side effects induced by breast cancer therapy and other gynecological issues, such as fertility preservation or contraception, which need to be addressed differently in breast cancer patients. Previously, these contents could be found as part of various other chapters.

\section{Menopausal Symptoms}

Classical hormonal therapy to alleviate menopausal symptoms is not indicated in breast cancer patients (particularly in ER-positive disease) but might be considered in individual cases and after failure of other non-hormonal treatments (LoE 2a/B/AGO +). Tibolone is contraindicated [96] ( $\mathrm{LoE} 1 \mathrm{~b} / \mathrm{A} / \mathrm{AGO}-$ ), while topical vaginal application of estriol may be used for urogenital symptoms [97] (LoE 4/D/AGO +/-). Climacteric symptoms such as hot flashes, night sweats, or sleep disturbances may be treated with various non-hormonal remedies, e.g. serotonin reuptake inhibitors (i.e. venlafaxine $(\mathrm{LoE} 1 \mathrm{a} / \mathrm{A} / \mathrm{AGO}+))$ or gabapentin $(\mathrm{LoE} 1 \mathrm{a} / \mathrm{A} / \mathrm{AGO}+)$, which carry the potential to reduce hot flashes by about $60 \%$ [98].

The majority of studies regarding the efficacy of herbal treatments for menopausal symptoms - mostly hot flashes - have not been conducted in women with breast cancer and many are of short duration [99]. Increased pharmacovigilance for herbal medicines is required, e.g. initiatives to stimulate reporting of suspected adverse reactions. Overall, red clover users were less likely to report weight gain, night sweats, and difficulty concentrating [100]. Soy-derived isoflavonoids are potent phytoestrogens, which can interact with ERs, and their dose-response relationships with ERs in vitro are complicated [101]. Flaxseed has no effect with regard to reducing hot flashes, based on a randomized phase III trial where it failed to demonstrate a significant reduction of hot flashes in postmenopausal patients taking an additional $410 \mathrm{~g}$ of lignans as compared to placebo [102]. In a phase III trial, the fixed combination of red clover and St. John's wort was significantly better in reducing menopausal symptoms compared to placebo. In a recent randomized placebocontrolled trial in 72 women without breast cancer suffering from hot flashes, $40 \mathrm{mg}$ red clover leaves showed a significant reduction of hot flashes based on the menopausal rating scale compared to placebo [103]. Taken together, neither black cohosh (Cimicifuga racemosa) [104] nor St. John's wort [105] nor Dong Quai (Zhuang SR) nor ginseng root [106] could improve menopausal symptoms.

\section{Fertility Preservation}

Counseling on fertility preservation is suggested in all patients who need to preserve their fertility ( $\mathrm{LoE} 4 / \mathrm{c} / \mathrm{AGO}+$ ). GnRH analogues can still not be considered as the treatment of choice, although the latest Prevention of Early Menopause Study (POEMS) showed a significantly higher rate of recovery of ovarian function after 2 years. However, in multivariable analysis, GnRH treatment was not an independent predictor of ovarian reserve [107] ( $\mathrm{LoE} 1 \mathrm{~b} / \mathrm{B} / \mathrm{AGO}+/-$ ).

The menstrual history is reliable only in women under 45 years of age. A more precise evaluation of the ovarian reserve (particularly in perimenopausal patients) may be obtained by the measure- ment of FSH and E2 levels in peripheral blood. Low anti-Muellerian hormone $(\mathrm{AMH})$ levels seem to be indicative of reduced ovarian reserve and chemotherapy-related amenorrhea (CRA) in chemotherapy-treated breast cancer patients. An antral follicle count, defined as the sum of the follicle diameters of all follicles of $10 \mathrm{~mm}$ in both ovaries, can easily be performed at little extra cost $(\mathrm{LoE} \mathrm{3b/B/AGO} \mathrm{+/-)} \mathrm{[108].}$

\section{Contraception}

All patients of childbearing potential must be counseled about adequate contraception during systemic therapy [109], since cytotoxic treatment by itself does not confer reliable protection against pregnancy. The majority of contraceptive measures have not been tested in women after breast cancer.

\section{Oncoplastic and Reconstructive Surgery}

Oncoplastic surgery is defined as the use of plastic surgical techniques at the time of tumor excision to enable safe resection margins and to preserve aesthetic breast contours. Oncoplastic surgery increases the number of breast-conserving therapies, enables the resection of larger tumors, reduces the number of re-excisions and leads to high patient satisfaction. Local RRs are the same as in classical breast-conserving therapy [110].

If breast-conserving therapy is not feasible, breast reconstruction with silicone-filled breast implants, free or pedicled autologous tissue transfer reconstruction, or autologous tissue transfer combined with implants should be offered ( $\mathrm{LoE} \mathrm{B} / \mathrm{AGO}+$ ) (fig. 5, $w w w . k a r g e r . c o m / ? D O I=431346)$. Indications for the various techniques must be weighed up carefully depending on the patients and tumor issues. In mastectomy, preservation of the nipple-areola complex may be performed ( $\mathrm{LoE} 2 \mathrm{~B} / \mathrm{B} / \mathrm{AGO}++$ ). The best implant reconstruction (IR) results will be achieved if no locoregional irradiation will be necessary (LoE A/AGO ++). In case of need for radiotherapy, IR prior to radiotherapy should be preferred (LoE 2a/ $\mathrm{AGO}+)$ as compared to IR following mastectomy and radiotherapy (LoE B/AGO +/-). Nevertheless, complications like capsular contraction, necrosis, infections etc. in at least $40 \%$ of the cases have been described. Synthetic meshes or acellular dermal matrices (ADMs) are possible options for muscle fixation in case of immediate implant reconstruction ( $\mathrm{LoE} 2 \mathrm{~b} / \mathrm{C} / \mathrm{AGO}+$ ).

If autologous reconstruction is planned (transverse rectus abdominis muscle (TRAM), deep inferior epigastric perforator (DIEP)), radiotherapy should be performed prior to reconstructive surgery in order to avoid higher rates of fibrosis and necrosis and poorer aesthetic results (LoE 2/AGO +) [111, 112].

The use of lipotransfer is an increasingly employed additional tool to refine breast-reconstructive surgery, with yet no data suggesting an increased risk of disease recurrence. Lipotransfer can be performed after mastectomy and implant-based reconstruction $($ LoE $2 \mathrm{a} / \mathrm{B} / \mathrm{AGO}+)$. After BCS, lipotransfer should only be per- 
formed on an individual basis and after detailed informed consent, due to lack of data (LoE 4/D/AGO +/-).

If implant reconstruction is not suitable, the preferred pedicled TRAM (LoE 3b/AGO +) or the free tissue flap DIEP (LoE 3b/AGO +) may be considered. Comparing both techniques on a data basis without prospective trials, the free tissue transfer is a more timeand personnel-consuming microsurgical procedure associated with a higher rate of reoperations, a higher total failure rate, and no increased patient satisfaction in multivariate analyses. Therefore, ipsilateral pedicled TRAM is recommended (LoE 3b/AGO +; fig. 5, www.karger.com/?DOI=431346) [113].

\section{Complementary Therapy - Survivorship}

This year, we focused on updating LoEs and grades of clinical recommendation of existing AGO slides in this chapter, since only little completely new information could be extracted from the literature. As before, Complementary and Alternative Medicine (CAM) treatments are scrutinized under 2 separate aspects: (1) improvement or amelioration of side effects of conventional treatment and (2) improvement of cancer-related outcome.

We would like to stress the fundamental considerations with regard to CAM treatment that we look upon as indispensable (fig. 6, www.karger.com/?DOI=431346).

Post-diagnosis Ginseng use did not improve the quality of life (QoL) of breast cancer survivors. Post-diagnosis Ganoderma lucidum use was associated with better social well-being scores, but poorer physical well-being scores [114]. A spice from India, curcumin, appears interesting in many respects. It was demonstrated by Ryan et al. that oral curcumin, $6.0 \mathrm{~g}$ daily during radiotherapy reduced the severity of radiation dermatitis in breast cancer patients [115].

Acknowledging the patients' needs to contribute to the treatment of their breast cancer, the recommendation to quit cigarette smoking has been emphasized more clearly as compared to previous years [116]. Furthermore, the necessity to adhere to a healthy nutritional and dietary concept according to recognized food recommendations is emphasized. Overall, a reduction of fat uptake pays off in various health aspects and is more generally recommended.. One important link between reduced fat intake and disease-specific as well as general health effects is the reduction of obesity, which clearly improves OS and DFS [117]. More specifically, newer data suggest that high-fat dairy products should be avoided [118]. Notably, this year, we lifted the ban on some complementary treatments for the prevention of recurrence that widely ruled previous editions. Scientific evidence suggests that (i) vitamin and/or antioxidant supplements do not jeopardize disease outcomes and (ii) particularly vitamins C, E and D might hold some beneficial effects [119-121], while artificial carotenoids should be viewed with caution [122]. There are no reliable data on which to refute the wish of patients to add moderate amounts of soy products to their food plans. However, soy concentrates containing an excess of $100 \mathrm{mg}$ of isoflavonoids per day will have to prove their safety before their use can be recommended [123]. Current evidence does not support an association between the intake of black cohosh and an increased risk of breast cancer [124]. For acupuncture, evidence of benefit was found concerning cancer treatment-related nausea and vomiting. Benefit was also reported for other cancer-related symptoms, including pain, fatigue, hot flushes, xerostomia, dyspnea, and anxiety. Reviewers found a paucity of rigorous trials and heterogeneity of populations, interventions, controls, and outcome measures, which challenge the process of systematic review and meta-analysis.

Acupuncture should be considered for symptom management where there are limited treatment options, using current peer-reviewed guidelines and clinical reasoning [125]. Mindfulness-based stress reduction (MBSR) is an 8-week program aiming at developing participants' coping resources and mindful awareness. The program consists of guided meditations, guided body scan (a specific awareness exercise) and meditation, and yoga. Psychoeducation tackles stress and stress reactions. Reviews and meta-analyses on breast cancer patients showed positive effects on their mental health status. The meta-analysis by Cramer et al. [126] including only randomized controlled trials reported small effects on depression and moderate effects on anxiety.

\section{Supplemental Figures}

Fig. 1. Strategy after diagnosis of central papilloma.

Fig. 2. Surgical treatment of axillary lymph nodes pre and post NACT.

Fig. 3. Axillary interventions in patients with positive sentinel lymph nodes.

Fig. 4. Adjuvant chemotherapy without concurrent trastuzumab.

Fig. 5. Postmastectomy reconstruction.

Fig. 6. Fundamental considerations in integrative oncology.

To access the supplemental figures, please refer to www.karger.com/? $D O I=431346$.

\section{Disclosure Statement}

C.L. has received consultant and speaker honoraria from Amgen, Celgene, Genomic Health, GSK, Novartis, Pierre-Fabre, Roche, and TEVA. M.T. has received consultant and speaker honoraria from AstraZeneca, Amgen, Celgene, Genomic Health, GSK, Novartis, Pierre-Fabre, Serag-Wiessner, Roche, and Sysmex Europe. V.H. has received consultant and speaker honoraria from Novartis and Roche. F.S. has received consultant and speaker honoraria from AstraZeneca, Amgen, Celgene, Novartis, Roche Pharma, and Medtronic. The authors have no relevant affiliation or financial involvement with any organization or entity with financial interest in or financial conflict with the subject matter or materials discussed in the manuscript apart from the disclosed.

\section{References}

References can be found in the appendix, please refer to

www.karger.com $/$ ?DOI $=431346$. 